\section{Distance discrimination in a reduced cue setting 1}

JOHNNY K. WORLEY and ROBERT $P$. MARKLEY, Texas Christian University, Fort Worth, Tex. 76129

Five Ss made nearer-farther distance discriminations in a reduced cue setting simulating outer space. The results confirm previous findings that the Weber function for apparent distance is a power function, with an exponent greater than 1.

In the typical investigation of distance discrimination, judgments are potentially influenced both by cues associated with the stimulus itself (e.g., accommodation, convergence, retinal disparity, and retinal image size) and by situational cues (texture gradients, aerial perspective, superposition, etc.) that are not necessarily stimulus specific. There are real situations in which the latter types of cues are missing. One example would be an $O$ located in outer space. However, recent research has indicated that human Ss can make distance judgments and discriminations in the absence of terrain and textural cues with reasonable accuracy (Vincent, Brown, Markley, \& Arnoult, 1969; Kunnapas, 1968; Markley, Brown, \& Arnoult, 1969).

The scaling study by Künnapas (1968) examined several visual cues to distance perception. Retinal image size alone provided nearly the same depth-judgment results as unrestricted viewing. In Künnapas's experiment, small targets and a distance range of only 0.25 to $3.95 \mathrm{~m}$ were used. Vincent et al (1969) studied distance discrimination of a large target over long ranges (up to $12,800 \mathrm{ft}$ ) in a reduced cue situation simulating outer space. The stimulus object was a representation of the Apollo Command and Service Module (CSM). Their results supported the notion that the Weber function relating a just noticeable change in distance to distance $(\Delta D$ to $D)$ was a power function, with an exponent greater than 1.0. Teichner, Kubrick, \& Disek (1955) had earlier summarized several studies and reported an exponent of 1.35. The exponents from the Vincent et al data were 1.19 and 1.20 for proximal (change toward the $S$ ) and distal (change away from $S$ ) Weber functions, respectively. The actual $\Delta D$ s obtained by Vincent et al (1969) were only slightly larger than those reported by Teichner et al (see Fig. 3 of Vincent et al, 1969). The reduced cue situation apparently did not seriously reduce discriminability of stimulus distance. Vincent et al (1969) reported that retinal image size was probably the most important cue to depth discrimination in their experiment. They discussed the possibility of other visual cues (binocular disparity, light-ray configurations, convergence or accommodation changes) contributing to the Ss' judgments, but the importance of these cues was probably not great due to the large distances used.

The present study was designed to replicate the Vincent et al (1969) experiment, using a less familiar and more complex stimulus object differing in size and overall configuration.

\section{SUBJECTS}

Five male undergraduates from Texas Christian University were paid to participate in this experiment. Two Ss were AFROTC pilot trainees, and all possessed uncorrected $20 / 20$ visual acuity in both eyes. PROCEDURE

The method of constant stimuli was used to obtain proximal and distal $\Delta D$ values for each of four standard distances. Standard and comparison distances were presented sequentially. The standard distances were $400,900,2,100$, and $3,200 \mathrm{ft}$. Five comparison distances were paired with each standard. Each $S$ judged every standard-comparison pair 20 times. The criteria for selecting the comparison distances and the procedures followed in presenting stimulus pairs and obtaining judgments were in all other aspects the same as described by Vincent et al (1969).

\section{APPARATUS}

The NASA-TCU Space Vision Simulator was used in this experiment. The simulator allows three-dimensional presentation of an object (in this experiment, a model of the ascent stage of the Lunar Module, LM) in a star-free outer-space setting. The optomechanical simulator is capable of presenting objects at distances ranging from 200 to $20,000 \mathrm{ft}$. Appropriate retinal image sizes, binocular cues, light-ray configuration, and relative brightness changes are maintained. A detailed description of the simulator has been reported elsewhere (Arnoult, Vincent, Brown, Markley, \& Hensleigh, 1968). The ascent stage of the LM is a complex, irregularly shaped object and, at the time, unfamiliar to the Ss. The orientation of the LM was fixed and slightly tilted to the left and away from the Ss. The simulated visible dimensions of the LM in this attitude were approximately $14 \mathrm{ft}$ wide at its base, $8.5 \mathrm{ft}$ wide at the top portion, and $9 \mathrm{ft}$ tall.



Fig. 1. Proximal and distal thresholds for distance as a function of observation distance.

\section{RESULTS}

Distal and proximal thresholds $(\Delta D)$ were obtained in the manner described by Vincent et al (1969). These results are shown in Fig. 1. The open circles and triangles in Fig. 1 represent part of the data previously obtained by Vincent et al (1969). The present data were adequately described by a power function

$$
\Delta \mathrm{D}=0.0076 \mathrm{D}^{1.27}
$$

determined by the method of least squares. The regression line accounts for $98.2 \%$ of the variance of the dependent variable. Functions fitted to proximal and distal $\Delta D s$ separately were

$$
\begin{aligned}
& \Delta \mathrm{D}_{\mathrm{p}}=0.0062 \mathrm{D}^{1.29} \\
& \Delta \mathrm{D}_{\mathrm{d}}=0.0088 \mathrm{D}^{1.26}
\end{aligned}
$$

It can be seen that the exponents lie between those reported earlier by Vincent et al (1969) and the exponent reported by Teichner et al (1955). Additionally, Fig. 1 shows that the $\Delta D$ values (both proximal and distal) obtained in this study were consistently greater than in the previous study. The values of $\triangle D / D$ were also larger, varying from about $4 \%$ at $400 \mathrm{ft}$ to $7 \%$ at $3,200 \mathrm{ft}$. Unlike the previous results, all $\Delta D_{p}$ values were smaller than the $\Delta D_{d}$ values for the same standard.

DISCUSSION

The results of this study support previous findings that the Weber function for distance is a power function with an exponent greater than 1.0. As can be seen in Fig. 1, both proximal and distal $\Delta D$ values 
were consistently greater than those obtained by Vincent et al (1969), but the slopes of the lines fitting the two sets of data were quite similar. Considering the differences in stimulus objects used, these results tend to corroborate those of the previous study. The question of the relative importance of various cues in distance discrimination is not answered by these data. The data indicate that, in the absence of terrain information, Ss are capable of making reasonably precise distance discriminations. Retinal image size probably makes the most significant contribution to this accuracy.

In view of the greater $\Delta \mathrm{D}$ values obtained in this study and the relatively large degree of between-S variability, additional data were collected using the same method and procedure described above for one of the Ss whose responses were most consistent. One hundred judgments were made for each of seven comparison-standard pairs. The standard distance used was $400 \mathrm{ft}$. The proximal and distal values of $\triangle D / D$ were $2.0 \%$ and $2.25 \%$, respectively, which are somewhat smaller than those reported above. These data indicate that $O$ sensitivity and experience are critical in determining $\Delta \mathrm{D}$ values, particularly for the tasks described earlier, but over a range of standard distances, the power function describing distance discrimination would not be significantly affected.

The results obtained from these reduced cue studies and the well documented usefulness of textural and context cues lends indirect support to Ross \& DiLollo's (1968) recent suggestion that $S s$ can and do shift the relative importance of particular stimulus attributes so as to maximize their ability to discriminate in the experimental situation.

\section{REFERENCES}

ARNOULT, M. D., VINCENT, R. J., BROWN, B. R., MARKLEY, R.P., \& HENSLEIGH, R.C.A description of the NASA-TCU space vision simulator. Technical Report, September 1968, Texas Christian University, Grant NGR 44009 018, National Aeronautics and Space Administration, CR 73305.

KUNNAPAS, $T$. Distance perception as a function of available visual cues. Journal of Experimental Psychology, 1968, 77, 523-529.

MARKLEY, R. P., BROWN, B. R., \& ARNOULT, M. D. Fractionation of distance in simulated space. Technical Report, January 1969, Texas Christian University, Grant NGR 44009018 . National Aeronautics and Space Administration, CR 73306.

ROSS, J., \& DiloLlo, V. A vector model for psychophysical judgment. Journal of Experimental Psychology, 1968, 77, (Monograph Supplement).

TEICHNER, W. H., KUBRICK, T. L., \& DISEK, E. $R$. Commonplace viewing and depth discrimination. Journal of the Optical Society of America, 1955, 45, 913-920.

VINCENT, R. J., BROWN, B. R.; MARKLEY, R. P., \& AR NOULT, M.D. Distance discrimination in a simulated space environment. Perception \& Psychophysics, 1969, 5, 235-238.

$$
\text { NOTE }
$$

1. This research and preparation of this paper was supported by Grant NGR 44009018 from the National Aeronautics and Space Administration to Texas Christian University; $M$ D. Arnoult was the principal investigator.

\section{Persistence of GSR changes as a function of repeated verbal satiation of emotionally-charged words}

\section{NICHOLAS S. DiCAPRIO and MARY-THOMAS TURNOCK, John Carroll University, Cleveland, Ohio 44118}

The effects of repeated verbal-satiation applications, i.e., rapid repetitions of words for several minutes at a time, was studied over the course of several weeks by means of GSR changes. Highly charged, personally relevant words were divided into experimental words and control words, equally matched on emotional-response intensity. The satiation treatment consisted of three 40-min periods occurring once a week, with the outcome that the formerly highly charged words that were satiated yielded significantly lower GSR tracings than did the highly charged but unsatiated words. The satiation effect persisted for at least a week, i.e., the time lapse between the last satiation session and the post-satiation test. GSR levels dropped more quickly after the first satiation, and continued to drop throughout the rest of the satiation sessions. Thus, the interpretation of the effects of reactive inhibition as the sole process involved in satiation was questioned.

One of the most characteristically human psychological functions involves the use of language symbols. Such language symbols acquire "meaning" through learning processes. The "real meaning" of a word, however, is ultimately a highly personal matter. Prolonged visual fixation of words, or long-continued repetitions of "emotionally charged" words, will produce change or loss of meaning of the words, i.e., a satiation effect. The loss or change of response may be thought of in two ways: the associative potential or cognitive aspect of the words, and the connotative aspect that includes the physiological reactions to the stimuli.

Severance \& Washburn (1907), using Ss' reports of loss of meaning of words that were visually fixated for several minutes, ascribed the satiation effects to oscillation of attention and a subsequent bre akdown of the stimulus words into "meaningless" units. The phenomenon of satiation was described by them as temporary. For the most part, this view has prevailed, even though later investigators have refined the concepts and methods of satiation considerably. For example, the loss of cognitive aspects of words has been measured by response latency (Baras, 1968), rareness of associations suggested by satiated words (Smith \& Raygor, 1956; Fillenbaum, 1963), and the number of associations that are suggested in a given period of time (Noble, 1952, Fillenbaum, 1963; Baras, 1968). The connotative aspect of words has been measured by use of the Osgood semantic-differential scales (Lambert \& Jakobovits, 1960). Mason (1941), using GSR changes in a preliminary study of satiation effects, demonstrated that GSR records can take the place of introspective reports as an indicator of satiation effects.

The purpose of the present study was to determine the extent to which emotional responses elicited by personally relevant verbal stimuli, and measured by GSR tracings as the indicator of satiation, could be reduced by the application of the satiation procedures. The hypothesis is that repeated application of satiation procedures will yield decreased and enduring GSR levels.

METHOD
Ten students taken from an upper-division psychology course, taught during a 5-week summer session, participated as $S s$ in the experiment. Each $S$ came once a week for 5 weeks according to the following schedule: a pre-satiation test session the 1st week, followed by three satiation sessions in the subsequent 3 weeks, and concluded with a final post-satiation test period the 5 th week. Ss were asked to list 20 personally relevant and 20 neutral words prior to testing procedures. To assist the Ss in identifying personally relevant words, both written and oral instructions were given with examples of such categories of sensitivity as names, places, dates, etc. The words were to be rated on a 5-point scale of intensity; this was done not only to aid the Ss, but also later to match the words with corresponding GSR levels. It should be noted that the words listed did not always produce GSR levels that matched the Ss' ratings of them (i.e., sometimes words 